[0212-7199 (2005) 22: 6; pp 275-278] ANALES DE MEDICINA INTERNA Copyright $(2005$ ARAN EDICIONES, S.L

AN. MED. INTERNA (Madrid) Vol. 22, N. ${ }^{\circ} 6$, pp. 275-278, 2005

\title{
Pericardiocentesis en una Unidad de Cuidados Intensivos
}

\author{
R. RIDRUEJO SÁEZ, B. ZALBA ETAYO \\ Unidad de Cuidados Intensivos. Hospital Clínico Universitario Lozano Blesa. Zaragoza
}

\author{
PERICARDIOCENTESIS IN A CRITICAL CARE UNIT
}

\section{RESUMEN}

Objetivo: Estudiar un grupo de pacientes a los que se realizó pericardiocentesis valorando etiología del derrame pericárdico, complicaciones de la técnica y mortalidad.

Método: 69 pacientes ingresados en UCI del Hospital Clínico de Zaragoza diagnosticados de derrame pericárdico subsidiario de pericardiocentesis

Resultados: Presentamos una serie de 69 pacientes con edades comprendidas entre 20 y 87 años; 21 mujeres y 48 varones. La etiología más frecuente del derrame fue neoplásica en 19 casos $(27,5 \%)$, con predominio de cáncer de pulmón y de cáncer de mama. 17 casos $(24,6 \%)$ por iatrogenia y 14 (20,3\%) idiopática. El diagnóstico se realizó en el 63,8\%, 44 casos, previamente a su llegada a UCI. La realización del drenaje por punción se llevó a cabo en las primeras 12 h en 40 casos $(58 \%)$ siendo superior a $500 \mathrm{cc}$ en $37(53,6 \%)$. Se registraron complicaciones de la técnica en 7 casos $(10,3 \%)$. Fallecieron 16 pacientes $(23 \%)$, el $45 \%$ en las 12 primeras horas. Hubo más éxitus entre los pacientes con derrame pericárdico de causa mecánica.

Conclusión: Comentar que la etiológia del derrame pericárdico ha cambiado en los últimos años, el diagnóstico es sencillo y mínimas las complicaciones al evacuarlo, ya que los medios y monitorización actual permiten realizar la técnica en óptimas condiciones.

PALABRAS CLAVE: Derrame pericárdico. Taponamiento cardíaco. Pericardiocentesis.

\begin{abstract}
Objective: Study pacients diagnosed of pericardial effusion and pericardiocentesis was made. We search the ethiology, complications and thecnic's mortality.

Method: 69 patients of Clinic Hospital of Zaragoza admited in the cricital care unit.

Results: We present 69 patients with 20-87 years old, 21 women and 48 men. The most frecuently ethiology was neoplasic, 19 patients $(27.5 \%)$, in most cases lung and breast cancer; 17 (24.6\%) yatrogenic and 14 (20.3\%) idiopatic. Diagnosis was made previously at ICU in $63.8 \%$ (44 patients). Pericardiocentesis was made in the 12 hours after admission in 40 cases (58\%), and was superior than 500cc in 37 $(53.6 \%)$. We registered pericardiocentesis complications in $7(10.3 \%)$. 16 patients dead (23\%) most of them with pericardial effusion by mecanic cause.

Conclusion: Was the pericardial effusion etiology has changed last years, the diagnosis is simply and minimun complications in evacuation, because the means and actual monitorization allow to make pericardiocentesis in optimun conditions.
\end{abstract}

KEY WORDS: Pericardial efusión. Cardiac tamponade. Pericardiocentesis.

Ridruejo Sáez, R, Zalba Etayo B.Pericardiocentesis en una Unidad de Cuidados Intensivos. An Med Interna (Madrid) 2005; 22: $275-278$.

\section{INTRODUCCIÓN}

El derrame pericárdico es el resultado de un acúmulo de líquido que excede la posibilidad de drenaje del mismo; puede tratarse de exudado seroso, sangre, pus o gas que ocupa el saco pericárdico aumentando progresivamente la presión intrapericárdica. Cuando la presencia de este derrame ocasiona taquicardia, hipotensión, pulso paradójico y elevación de la presión venosa central, nos encontramos ante un taponamien- to cardíaco. Si la instauración del mismo ha sido lenta, su tolerancia clínica y hemodinámica será mejor, en caso de que sea rápida, pequeñas cantidades de líquido pueden ocasionar graves consecuencias. El diagnóstico se basa principalmente en la ecocardiografía que mostrará colapso diastólico de cavidades derechas, ocasionalmente de aurícula izquierda y rara vez de ventrículo izquierdo $(1,2)$. Puede haber falsos positivos por hipovolemia y falsos negativos por rigidez de cavidades debido a hipertrofia y enfermedad valvular, especialmente insufi-

Trabajo aceptado: 25 de febrero de 2005

Correspondencia: Raquel Ridruejo Sáez. Unidad de Cuidados Intensivos. Hospital Clínico Universitario Lozano Blesa. C/ Alemania, 6, $3^{\circ}$ A. 50010 Zaragoza. 
ciencia aórtica (3). El eco-Doppler detecta la variación de los flujos transvalvulares con la respiración, siendo una prueba con alta especificidad (4).

La pericardiocentesis es la técnica de elección utilizada para el drenaje del líquido, que se realizará de forma urgente o programada, según la situación hemodinámica del paciente. En el caso de derrames crónicos la indicación de pericardiocentesis diagnóstica no está claramente establecida.

Nuestro objetivo consiste en estudiar un grupo de pacientes a los que se realizó pericardiocentesis valorando etiología del derrame pericárdico, complicaciones de la técnica y mortalidad.

\section{PACIENTES Y MÉTODO}

Se han obtenido los datos correspondientes a 69 pacientes ingresados en la Unidad Médica de Cuidados Intensivos del Hospital Clínico Universitario de Zaragoza, durante el periodo de enero de 1995 a marzo de 2004. Se trata de un Hospital terciario de 800 camas que incluye los servicios de cardiología, oncología, medicina interna y nefrología como fuentes de ingresos por derrame pericárdico en nuestra serie; no disponemos de cirugía cardíaca por lo que no se recogen casos en relación con este origen.

El criterio de inclusión fue existencia de derrame pericárdico significativo que requirió pericardiocentesis. Se investigaron de forma retrospectiva las historias clínicas de pacientes que presentaban derrame pericárdico entre los diagnósticos al alta, descartando aquellos en los cuales no se llevó a cabo pericardiocentesis bien por no ser significativo o porque el paciente no sobrevivió el tiempo suficiente.

A todos ellos se realizó anamnesis, exploración clínica, radiografía de tórax, bioquímica, hemograma, estudio de coagulación y ecocardiograma.

De cada paciente se recogieron las siguientes variables: antecedentes personales, edad, sexo, factores de riesgo cardiovascular, estancia en UCI, momento del diagnóstico, situación hemodinámica al ingreso, necesidad de drogas vasoactivas, pruebas diagnósticas realizadas, momento de la pericardiocentesis, característiscas y cantidad de líquido, etiología, tiempo del drenaje, complicaciones, exitus.

El diagnóstico etiológico del taponamiento se basó en los datos de la historia clínica y la evolución posterior así como los datos bioquímico-microbiológicos del líquido en los casos en los que se disponía de ellos.

Los datos se registraron en Excell y para el tratamiento estadístico utilizamos el programa SPSS. Se obtuvo las medias y desviaciones estándar de las variables cuantitativas y los porcentajes de las cualitativas. Para la comparación de porcentajes entre grupos se utilizó el test de Chi cuadrado, el nivel de significación se estableció con una $\mathrm{p}<0,005$.

\section{RESULTADOS}

Presentamos una serie de 69 pacientes con edades comprendidas entre 20 y 87 años con una media de $64,1 \pm 15,1$. De ellos, $21(30,4 \%)$ eran mujeres y 48 varones $(69,6 \%)$. Entre los antecedentes personales encontramos hipertensión en 24 casos $(34,7 \%)$, diabetes mellitus en 7 casos $(10 \%)$, dislipemia en $14(20,3 \%)$, obesidad 3 (4,3\%), tabaquismo 19
(27,5\%), cardiopatía isquémica $16(24,6 \%) .24$ pacientes del total $(34,7 \%)$ tenían uno o más factores de riesgo cardiovascular.

La etiología más frecuente del derrame pericárdico en nuestra serie fue la neoplásica en 19 casos $(27,5 \%)$, con predominio de cáncer de pulmón, seguido en frecuencia de cáncer de mama. En segundo lugar, 17 casos $(24,6 \%)$ por iatrogenia incluyendo perforaciones coronarias en relación a estudios hemodinámicos y lesiones miocárdicas tras colocación de marcapasos, tanto provisionales como definitivos. La siguiente etiología en frecuencia fue la idiopática con 14 casos (20,3\%), seguida de causas mecánicas en 9 casos (13\%) que abarca disección aórtica en 4 ocasiones y 5 roturas cardíacas postinfarto de miocardio. A continuación registramos 7 casos $(10 \%)$ de etiología infecciosa y por último otros 3 casos $(4,3 \%)$ de origen urémico.

En el momento del ingreso presentaron insuficiencia cardíaca 59 pacientes $(85,5) \%$, disnea 50 pacientes $(72,4 \%)$ y dolor torácico 14 (20,2\%).

Ingresaron procedentes de planta de hospitalización 30 pacientes (43,5\%), 17 del servicio de Urgencias (24,6\%), 12 del servicio de hemodinámica $(17,4 \%), 8$ trasladados de otro centro sanitario $(16 \%)$ y de quirófano 2 pacientes $(2,9 \%)$. Observamos que los pacientes con etiología tumoral procedían con más frecuencia de planta de hospitalización, de éstos, el $100 \%$ habían sido diagnosticados antes del ingreso en UCI.

Más de la mitad (60\%) mantenían sus constantes estables en el momento del ingreso, precisando un $30 \%$ del total de nuestros pacientes drogas vasoactivas durante su evolución.

El diagnóstico se realizó en el 63,8\%, 44 casos, previamente a su llegada a la Unidad y en $25(36,2 \%)$ durante su estancia en la misma.

La realización del drenaje por punción se realizó en las primeras $12 \mathrm{~h}$ en 40 casos (58\%), entre 12 y 24 h en 9 (13\%) y después de 24 h en 20 (29\%). La vías de acceso fue subxifoidea en 68 casos y en 1 paraesternal izquierdo.

Las características del líquido drenado fueron: hemático en $52(75,4 \%)$, sero-hemático $15(21,7 \%)$ y purulento en 2 (2,9\%). Se obtuvo la siguiente cantidad: menos de $250 \mathrm{cc}$ en 14 pacientes $(20,2 \%), 18$ entre $250-500$ cc $(26 \%)$ y más de 500 cc en $37(53,6 \%)$.

El drenaje fue sólo evacuador en 19 ocasiones (27,5\%,), retirándose el catéter antes de 24 horas en 24 pacientes $(34,8 \%)$ y dejándolo más de un día en $26(37,7 \%)$.

Encontramos derrame pleural asociado al pericárdico en 16 ocasiones $(23,2 \%)$, siendo más frecuente esta asociación cuando la etiología del derrame era tumoral (p 0,014).

Se registraron complicaciones de la técnica en 7 casos $(10,3 \%)$ que consistieron en obstrucción del catéter en 3 casos (4\%), malposición con recolocación en 2 casos $(3,9 \%)$, arritmias y extracción dificultosa 1 caso cada una $(1,4 \%)$.

Fallecieron 16 pacientes (23\%), de éstos, el $45 \%$ en las 12 primeras horas. Hubo más éxitus entre los pacientes con derrame pericárdico de causa mecánica siendo estadísticamente significativo (p 0,039), asi mismo, se observaron más éxitus cuando el líquido era hemático (p 0,043).

\section{DISCUSIÓN}

En los últimos tiempos hemos asistido a un incremento progresivo de técnicas diagnósticas dirigidas a conocer la 
etiología de distintos procesos. Es por esto que las causas o el orden de frecuencia de algunos de ellos han cambiado, como ocurre en la patología pericárdica. En ella la pericarditis aguda idiopática (5) descrita como causa más frecuente de taponamiento en las primeras series publicadas, ha sido sustituida en trabajos más recientes por etiologías más específicas, especialmente la neoplásica $(6,7)$. Este dato coincide con lo encontrado en nuestro estudio, siendo además el cáncer de pulmón el que con más frecuencia produce taponamiento,como refieren otros trabajos $(8,9)$.

Por otra parte, el aumento de técnicas diagnósticas y terapéuticas invasivas ha favorecido también un incremento de derrames secundarios a yatrogenia, que constituyen nuestra segunda etiología. En la literatura se describen una incidencia de hasta el 17,6\% de taponamientos asociados a cirugía cardiaca y siendo mucho más frecuente los asociados a cirugía valvular (entorno al 73\%) (10) que tras by-pass coronario (24\%). En nuestro hospital no disponemos de servicio de cirugía cardíaca por lo que carecemos de datos al respecto. Sí contamos con servicio de hemodinámica que realiza los cateterismos coronarios del área 3 de Aragón. La incidencia de perforación vascular durante o seguido a intervención coronaria percutánea es rara y oscila entre 0,2 y $0,6 \%$ (11-16) alcanzando el 0,8\% en algunas series (17) siendo más frecuente tras ateroablación que tras ACTP más Stent $(0,26$ vs $0,11 \%)$ según un estudio de más de 25.000 pacientes publicada por Fejka y cols. (18). Nosotros hemos registrado 10 casos secundarios a intervensionismo percutáneo que corresponden a una tasa de $0,16 \%$ concordante con lo publicado. También es pequeño el porcentaje de derrame pericárdico secundario a colocación de marcapasos, entorno al 0,57-0,9\% si es definitivo y algo superior, alrededor del 2\%, si es transitorio (19). En nuestra serie objetivamos 7 casos correspondientes a esta etiología, 3 por marcapasos definitivo y 4 provisionales, aunque no disponemos de los datos totales ya que no todos los marcapasos definitivos ingresan en UCI.

No se consiguió demostrar etiología del derrame en 14 ocasiones $(20,3 \%)$ lo cual concuerda con los artículos revisados en los que este porcentaje oscila entre el 10-33\% $(9,15,20,21)$.

El diagnóstico del derrame pericárdico puede ser difícil en ocasiones, ya que puede presentarse con multitud de síntomas inespecíficos como disnea, dolor torácico o insuficiencia cardíaca que también se pueden presentar en otras muchas entidades, de ahí la importancia del contexto clínico y las pruebas complementarias, especialmente la ecocardiografía, para favorecer una identificación precoz del cuadro. En nuestra serie 44 casos $(63,8 \%)$ se diagnosticaron antes de su ingreso en UCI. Este grupo incluye todos los pacientes con un tumor maligno y aquellos cuyos síntomas clínicos fueron muy sugerentes de esta entidad (algunos en el contexto de procedimientos invasivos). En el caso de los pacientes con patología tumoral el diagnóstico clínico es más difícil por la instauración generalmente lenta del derrame, sin embargo, se les suele realizar múltiples pruebas de imagen que detectan la presencia de líquido acumulado en el saco pericárdico subsidiario de drenaje. De estos datos surge la explicación de que la mayoría de las pericardiocentesis (40 del total, $58 \%$ ) se realizara en las primeras $12 \mathrm{~h}$ de la llegada a UCI. Solo en el 36,2\% (25 casos) el diagnóstico no se sospechó o no existía previamente, realizándose durante la estancia en UCI, hecho que coincide con otra serie publicada recientemente (9).

No hemos encontrado asociación entre el aspecto del líquido pericárdico y etiologías concretas en nuestra serie, ya que el líquido hemático es el más frecuentemente observado en todas los grupos etiológicos, no solo en las neoplasias y la tuberculosis como se describe clásicamente (21).

Tampoco obtuvimos datos estadísticamente significativos entre la cantidad de líquido extraído o la duración del drenaje y las distintas etiologías.

La pericardiocentesis no es una técnica exenta de riesgos, habiéndose comunicado complicaciones importantes en un porcentaje entre el 10 y el $25 \%$ cuando se realiza a ciegas (2326), siendo la mortalidad alrededor del $4 \%$ en alguna de las mejores series (27).

Estas cifras mejoran notablemente cuando la técnica se realiza bajo control ecográfico además de monitorización electrocardiográfica y de tensión arterial, que sería la situación ideal si se dispone de ello en el momento de la punción.

Nosotros hemos objetivado complicaciones de esta técnica en 7 ocasiones (10,3\%), todas ellas consideradas leves, sin fallecimientos secundarios a la realización de la misma.

La mortalidad registrada fue del $23 \%$ (16 pacientes) durante su ingreso hospitalario, lo cual está dentro de los límites establecidos en la literatura $(12-30 \%)(28,29)$. Casi la mitad de los éxitus se produjeron en las primeras 12 horas, lo cual se comprende al observar que la causa más frecuente de muerte fue la mecánica, fundamentalmente por rotura cardíaca, postIAM y disección aórtica (p 0,039).

Como conclusión comentar que la etiología del derrame pericárdico ha cambiado en los últimos años, existiendo todavía un porcentaje considerable que no se llega a descubrir; el diagnóstico del derrame es sencillo y precoz y mínimas las complicaciones al evacuarlo, ya que los medios y monitorización actual permiten realizar la técnica en óptimas condiciones.

\section{Bibliografía}

1. Spodick DH, Roldan AC. The patient with pericardial disease. In: Roldan AC, Abrams J, eds. Evaluation for the patient with heart disease: integrating the physical exam and echocardiography. Philadelpia, Pa: Williams and Wilkins; 2002: 339-364.

2. Reydel B, Spodick DH. Frequency and significance of chamber collapses during cardiac tamponade. Am Heart J 1990; 119: 1160-1163.

3. Spodick DH. Acute pericarditis: current concepts and practice. JAMA 2003; 289: 1150-1153.

4. Lindenberger M, Kjellberg M, Karlsson E. Pericardiocentesis guided by
2-D echocardiography: the method of choise for treatment of pericardial effusion. J Intern Med 2003; 253: 411-417.

5. Permanyer-Miralda G, Sagristá-Sauleda J, Soler-Soler J. Primary acute pericardial disease; a prospective series of 231 consecutive patients. Am J Cardiol 1985; 56: 623-30.

6. Gibbs CR, Watson RB, Singh SP, Lip GY. Management of pericardial effusion by drainage; a survey of 10 years experience in a city centre general hospital serving a multiracial population. Postgrad Med J 2000; 76: 809-13. 
7. Guberman BA, Fowler NO, Engel PJ. Cardiac tamponade in medical patients. Circulation 1981; 64: 633-640.

8. Oliver C, Marín F, Pineda J et al. ¿Debemos pensar en una etiología específica en pacientes con taponamiento cardíaco? Rev Esp Cardiol 2002; 55: 493-498.

9. Eroles G, Rondón F, Ferreiro C et al. Análisis de 73 casos de derrame pericárdico significativo. Rev Clin Esp 2004; 204: 125-30.

10. Kuvin JT, Harati NA, Pandian NG et al. Postoperative cardiac tamponade in the modern surgical era. Ann Thorac Surg 2002; 74: 11481153.

11. Gruberg L, Pinnow E, Flood R et al. Incidence, management and outcome of coronary artery perforation during percutaneous coronary intervention. Am J Cardiol 2000; 86: 680-682.

12. Von Sohsten R, Kopistansky C, Cohen M et al. Cardiac tamponade in the new device era: evaluation of 6999 consecutive percutaneous coronary interventions. Am Heart J 2000; 140: 279-83.

13. Kimbris D, Iskandrian AS, Goel I et al. Transluminal coronary angioplasty complicated by coronary artery perforation. Cathet Cardiovasc Diagn 1982; 8: 481-7.

14. Grollier $\mathrm{G}$, Bories $\mathrm{H}$, Commeau $\mathrm{P}$ et al. Coronary artery perforation during coronary angioplasty. Clin Cardiol 1986; 9: 27-29.

15. Topaz O, Cowley MJ, Vetrovec GW. Coronary artery perforation during angioplasty; angiographic detection and demostration of complete healing. Cathet Cardiovasc Diagn 1992; 27: 284-288.

16. Nassar H, Hasin Y, Gotsman MS. Cardiac tamponade following coronary arterial rupture during coronary angioplasty. Cathet Cardiovasc Diagn 1991; 23: 177-179.

17. Gunning MG, Williams IL, Jewitt DE, Shah AM et al. Coronary artery perforation during percutaneous intervention: incidence and outcome. Heart 2002; 88: 495-498.
18. Fejka M, Dixon SR, Sfian RD et al. Diagnosis, management and clinical outcome fo cardiac tamponade complicating percutaneous coronary intervention. Am J Cardiol 2002; 90: 1183-6.

19. Calvo JM, Fernández de Soria R, Del Aguila M et al. Hemopericardio con taponamiento debido a perforación cardíaca por marcapasos transitorio. Emergecias 2000; 12: 56-58.

20. Sagrista M, Permanyer G, Carballo J et al. Pericardial efusión in the elderly: A different disease?. Rev Esp Cardiol 2000; 53: 1432-1436.

21. Atar S, Chiu J, Forrester JS, Siegel R. Bloody pericardial effusion in patients with cardiac tamponade: is the cause cancerous tuberculous or iatrogenic in the 1990s?. Chest 1999; 116: 1564-9.

22. Bastian A, Meissner A, Lins M. Pericardiocentesis: diferential aspects of a common procedure. Intensive Care Med 2000; 26: 572-6.

23. Dosios T, Theakos N, Angouras D, Asimacopoulos P. Risk factors affecting the survival of patients with pericardial efusión submitted to subxiphoid pericardiostomy. Chest 2003; 124: 242-246.

24. Allen KB, Faber LP, Warren WH, Shaar CJ. Pericardial effusion: subxiphoid pericardiostomy versus percutaneous catheter drainage. Ann Thorac Surg 1999; 67: 437-440.

25. Laham Roger J, Cohen DJ, Kuntz Richard E et al. Pericardial effusion in patients with cancer: outcome with contemporany manegement strategies. Heart 1996; 75: 67-71.

26. Kridorian JG, Hancock eW. Pericardiocentesis. Am J Med 1978; 65: 808-814.

27. Kilpatrick ZM, Chapman CB. On pericardiocentesis. Am J Cardiol 1965; 16: 722-728.

28. Nugue O, Millaire A, Porte $\mathrm{H}$ et al. Pericardioscopy in the etiologíc diagnosis of pericardial efusión in 141 consecutive patients. Circulation 1996; 94: 1635-1641.

29. Spodick, DH. Acute cardiac tamponade. N Engl J Med 2003; 349: 684-690. 